\title{
A AERODINÂMICA DAS CONSOANTES NASAIS [M] E [N] DO PORTUGUẾS BRASILEIRO
}

\author{
THE AERODYNAMICS OF [M] AND [N] NASAL CONSONANTS \\ IN BRAZILIAN PORTUGUESE
}

\author{
Michele Gindri Vieira | Lattes | michelegindri@gmail.com \\ Centro Catarinense de Reabilitação | Secretaria de Estado da Saúde - SC \\ Izabel Christine Seara | Lattes | izabel.seara@ufsc.br \\ Universidade Federal de Santa Catarina $\mid \mathrm{CNPq}$
}

Resumo: Este estudo tem como objetivo uma caracterização aerodinâmica das consoantes nasais $[\mathrm{m}] \mathrm{e}[\mathrm{n}]$ do português brasileiro, com o uso de tecnologias computadorizadas e coleta simultânea de dados acústicos e aerodinâmicos, em uma perspectiva dinâmica de produção da fala. As gravações envolveram cinco adultos, com idades entre 25 e 52 anos, três do sexo feminino e dois do sexo masculino. $\mathrm{O}$ corpus incluiu 20 logatomas em frases-veículo, variando o contexto de tonicidade e o da vogal precedente. Os dados de fala foram coletados por meio da Estação EVA (Evaluation Vocale Assistée) e do piezoelétrico. Dos dados advindos da Estação EVA, foram investigadas configurações de curvas de fluxo aéreo oral (FAO) e de fluxo aéreo nasal (FAN); e dos dados advindos do piezoelétrico, foram analisados valores das curvas de FAN. Os resultados indicaram, para ambos os sexos, semelhanças entre as consoantes [m] e [n]: muito baixa amplitude nas curvas de FAO, indicando oclusão na passagem de ar pela cavidade oral; e curvas de FAN com configuração plana em sua extensão e com maior amplitude em relação às vogais adjacentes. Assim, o comportamento aerodinâmico do FAN na produção das consoantes [m] e [n] permitiu a inferência articulatória de que o gesto vélico dessas consoantes seja formado por três tempos: abertura, platô e fechamento. Ainda, foram confirmadas diferenças significativas entre parâmetros aerodinâmicos relativos às consoantes $[\mathrm{m}] \mathrm{e}[\mathrm{n}]$, quanto ao contexto de tonicidade e ao da vogal precedente. As características aerodinâmicas apontaram, portanto, para a natureza dinâmica das consoantes nasais do português brasileiro.

Palavras-chave: Consoantes nasais; Português brasileiro; Análise aerodinâmica; Perspectiva dinâmica. 
Abstract: The present study aims to describe the aerodynamics of the [m] and [n] nasal consonants of the Brazilian Portuguese, by using computer technology and simultaneous collection of both acoustic and aerodynamic data, under a dynamic perspective of speech production. Five adults were recorded; three females and two males, aged from 25 to 52 years. The corpus included 20 logatomes embedded in carrier sentences. The context of tonicity and the context of the preceding vowel varied. Speech data were collected through the EVA Station (Evaluation Vocale Assistée) and piezoelectric instruments. The analysis of the data collected through the EVA Station concerned the curve characteristics of the oral aerial flow (OAF) and those of the nasal aerial flow (NAF), while the analysis of data collected through the piezoelectric concerned the values of the NAF curves. The results indicated, for both male and female participants, similarities between [m] and [n] consonants: very low amplitude in the OAF curves, which indicated some occlusion in the passage of air through the oral cavity; and NAF curves with plain configuration and with greater amplitude in relation to the underlying vowels. Therefore, the aerodynamic behavior of NAF during the production of [m] and [n] allowed for articulatory interference that showed that the velic gesture of these consonants are composed of three tempos: opening, plateau and closing. Additionally, there were significant differences among the aerodynamic parameters related to the $[\mathrm{m}]$ and $[\mathrm{n}]$ consonants, regarding the contexts of tonicity and preceding vowel. Hence, the aerodynamic characteristics pointed to the dynamic nature of nasal consonants of Brazilian Portuguese.

Keywords: Nasal consonants; Brazilian Portuguese; Aerodynamic analysis; Dynamic perspective.

\section{Introdução}

Este estudo tem como foco a aerodinâmica das consoantes nasais [m] e [n] do português brasileiro $(\mathrm{PB})^{1}$. Os sons nasais consonantais são intrinsicamente dependentes de fatores anatômicos e funcionais envolvidos no acoplamento entre as cavidades oral e nasal. O fluxo aéreo oral (doravante FAO) e, principalmente, o fluxo aéreo nasal (doravante FAN), decorrentes da produção das consoantes nasais, conferem variabilidade aerodinâmica e acústica à produção dessas consoantes. Isso é consequência dos movimentos do complexo mecanismo neuromuscular, que promove uma variedade de graus de abertura do esfíncter velofaríngeo (formado pelo véu palatino e pelas paredes da faringe), dire-

\footnotetext{
${ }^{1} \mathrm{O}$ presente artigo apresenta resultados da tese de doutorado "Estudo acústico e aerodinâmico das consoantes nasais do português brasileiro: variedade de Florianópolis (VIEIRA, 2017)”.
} 
cionando a passagem do fluxo aéreo para as cavidades oral e nasal. Pode-se observar, na Figura 1, a anatomia da cavidade oral e do esfíncter velofaríngeo.

Figura 1. Anatomia da cavidade oral e do esfíncter velofaríngeo. As setas indicam o posicionamento do véu palatino nos movimentos de abaixamento (1) e de elevação (2)

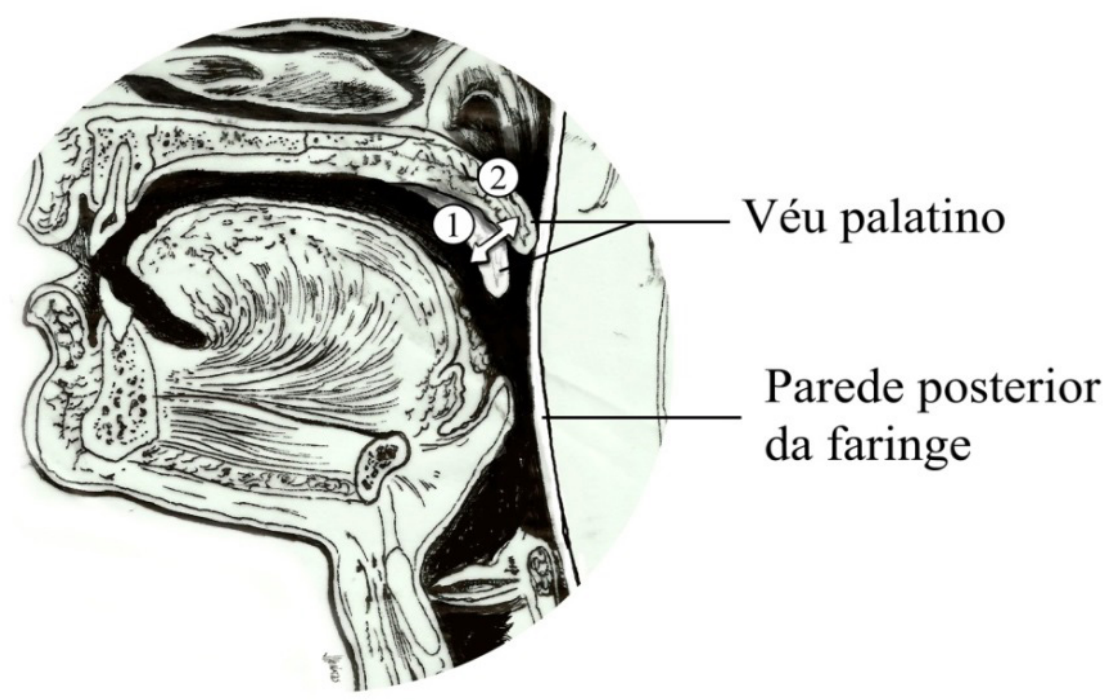

Fonte: Vieira (2017, p. 68).

As condições aerodinâmicas da fala são definidas pelo comportamento do trato vocal em relação à passagem do fluxo aéreo. Logo, a aerodinâmica estabelece uma relação intrínseca com os movimentos articulatórios e com a energia do sinal acústico oral, gerada a partir dos lábios, ou com o sinal acústico nasal, determinado a partir das narinas. Embora nem sempre seja percebido pelo ouvido humano, seu caráter acusticamente gradiente pode ser observado no detalhe fonético em dados do PB (VIEIRA, 2017).

Com o presente estudo, temos por objetivo ir além do aspecto acústico, apresentando uma caracterização aerodinâmica das consoantes nasais $[\mathrm{m}] \mathrm{e}[\mathrm{n}]$ do $\mathrm{PB}$, com o uso de tecnologias computadorizadas e coleta simultânea de dados acústicos e aerodinâmicos, complementada com correlatos articulatórios em uma perspectiva dinâmica de produção da fala.

Nossa hipótese inicial é a de que essas consoantes apresentem características aerodinâmicas semelhantes de FAO (sem saída de ar pela cavidade oral), uma vez que o bloqueio na cavidade oral ocorre na produção de ambos os sons ([m] e [n]), e de que se diferenciem em termos de FAN, o que se justificaria devido a pontos articulatórios distintos na cavidade oral, gerando consequente diferenciação no tamanho do tubo e na saída de ar pela cavidade nasal. 
Neste sentido, a questão a ser esclarecida é se, ao comparar as consoantes nasais [m] e [n], haveria distinção nos parâmetros do fluxo aéreo nasal entre esses sons, tais como: (i) tipos de configurações de curvas de FAN e (ii) valores de amplitude mínimos, médios, máximos, nos pontos inicial, medial e final, medidos nas curvas de FAN. Buscamos investigar, ainda, quais inferências articulatórias poderiam ser decorrentes dos resultados aerodinâmicos. Medições do FAN representam uma indicação indireta do grau de abertura velofaríngea, por existir uma correlação válida entre fluxo de ar e mecanismo velofaríngeo (VAISSIÈRE, 1995; AMELOT, MICHAUD, 2007; MEDEIROS, D’IMPERIO, ESPESSER, 2008). O estudo do FAN pode revelar, assim, movimentos articulatórios que não poderiam ser inferidos somente pelos dados acústicos.

Para tanto, apresentaremos uma descrição qualitativa das configurações das curvas de FAO e de FAN advindas dos dois instrumentos de análise aerodinâmica: Estação EVA e piezoelétrico. Faremos, ademais, uma análise quantitativa, com validação estatística de diferenças entre os valores mínimos, médios, máximos, do início, do meio e do fim da curva de FAN, advindos do piezoelétrico. Observaremos também a influência do contexto de tonicidade da sílaba em que a consoante se encontra e da qualidade da vogal precedente sobre os parâmetros aerodinâmicos (valores da curva de FAN). Analisaremos, pelo viés da perspectiva dinâmica da fala, a possibilidade de inferir aspectos articulatórios por meio de parâmetros aerodinâmicos.

Com base no exposto, o estudo justifica-se por contribuições à Linguística e a áreas do conhecimento correlatas, como a Fonoaudiologia, no que tange à análise acústica e aos mecanismos aerodinâmicos da produção da fala típica. Faz uso de ferramentas específicas, pouco utilizadas em pesquisas no país até o presente momento (Estação EVA e piezoelétrico), para descrever o comportamento aerodinâmico das consoantes nasais [m] e [n] do PB, associando-as a dados acústicos.

Na próxima seção, abordamos o referencial teórico com os principais pressupostos que embasam este artigo. A seguir, descrevemos a metodologia utilizada para a coleta dos dados acústicos e aerodinâmicos de fala. Na sequência, apresentamos uma seção com os resultados e a discussão sobre os dados. Por fim, na última seção, expomos nossas considerações finais.

\section{Referencial teórico}

O presente trabalho encontra respaldo em uma perspectiva dinâmica de produção da fala para investigar a natureza das consoantes nasais do português brasileiro. A Fonologia Gestual, implementada por Browman e Goldstein (1992), inicialmente cha- 
mada de Fonologia Articulatória, é fundamentada na premissa de que a variável tempo deve ser intrínseca aos modelos de análise fonológica (FOWLER, 1980). Assim, as noções de sistemas dinâmicos são inseridas na Linguística, tendo integradas a fonética e a fonologia que passam a constituir o nível fônico da língua.

Estudos deste tipo se enquadram ainda na vertente denominada de Fonologia de Laboratório (COHN; FOUGERON; HUFFMAN, 2012; SILVA; ALVES, 2016), que parte de dados empíricos obtidos a partir de análises acústicas e aerodinâmicas (levando à inferência articulatória) para embasar a representação de um fato fonológico. Portanto, a análise do dado fonético associada ao enfoque aerodinâmico permite conhecer as organizações temporais interligadas à produção de [m] e [n].

As consoantes nasais [m] e [n] no português europeu já foram estudadas sob o viés gestual por Oliveira e Teixeira (2007). Para a gravação dos dados articulatórios de fala de uma participante, foi usado um magnetômetro (AG100 EMMA). Essas autoras verificaram que o gesto do véu do palato para as consoantes nasais é formado por três tempos (abertura, platô e fechamento), confirmando a natureza dinâmica das nasais do português europeu, bem como a influência do contexto segmental no movimento velar. Por fim, concluíram que, em posição de onset, o fechamento oral está sincronizado ao abaixamento do véu palatino.

No Brasil, Mendonça e Seara (2015) estudaram sob o viés aerodinâmico as vogais nasalizadas coarticulatoriamente pelas consoantes [m] e [n] em sílabas adjacentes. Na coleta de dados, participaram dois adultos com fala típica e foi utilizado o piezoelétrico. As autoras verificaram uma diferença no surgimento da curva de FAN entre as consoantes nasais do PB, de modo que, para a produção de [m], a curva de FAN apresentou pouca antecipação sobre a vogal precedente, iniciando-se muito próximo do onset desse segmento; entretanto, para a produção do [n], a curva exibiu antecipação moderada.

O estudo de Marino et al. (2016) também traz contribuições sobre as diferenças nas características aerodinâmicas de sons nasais no PB. Nesse estudo, foi utilizado um nasômetro para análise de sílabas orais e nasais, produzidas por 245 falantes, de ambos os sexos, em quatro grupos (adultos, adultos jovens, adolescentes e crianças). Os resultados indicaram valores de nasalância mais altos para sílabas com sons nasais quando comparadas a sílabas com sons orais. E também, para a população adulta, valores mais altos de nasalância foram verificados para as mulheres quando comparados aos dos homens.

A seguir, serão elencados os procedimentos de coleta dos dados acústicos e aerodinâmicos de fala. 


\section{Metodologia}

Os dados coletados nesta pesquisa são de adultos que apresentavam fala típica, residentes na cidade de Florianópolis, estado de Santa Catarina (Brasil) ${ }^{2}$. As gravações envolveram cinco adultos, com idades entre 25 e 52 anos, três do sexo feminino e dois do sexo masculino, com fala típica florianopolitana (Santa Catarina/Brasil).

Os informantes consentiram sua participação e a disponibilidade dos dados para o Laboratório de Fonética Aplicada (FONAPLI) da Universidade Federal de Santa Catarina (UFSC), mediante assinatura do Termo de Consentimento Livre e Esclarecido (TCLE).

As consoantes $[\mathrm{m}]$ e $[\mathrm{n}]$, foco deste estudo, estavam inseridas em logatomas na posição de início de sílaba dentro da palavra (onset medial) dispostos em frases-veículo. Nesses logatomas, as consoantes nasais apareciam em contextos átono (pós-tônico) e tônico e eram precedidas por diferentes vogais. O corpus incluiu 20 logatomas paroxítonos trissilábicos em frases-veículo, conforme apresentado no Quadro 1.

Quadro 1. Corpus contendo os logatomas em frases-veículo por consoante nasal

$$
([\mathrm{m}] \text { e }[\mathrm{n}]) \text { do PB }
$$

Transcrição ortográfica das frases-veículo com pares de logatomas

\section{Transcrição codificada dos logatomas para textgrid}

\section{Consoante nasal bilabial}

\begin{tabular}{|c|c|c|c|}
\hline $\mathbf{l}$ & Digo papama pamapa baixinho. & [рарT6 m6 pacmTap6] & [pa'pëme pétmape] \\
\hline 2 & Digo papema pemapa baixinho. & [papTe $\sim$ m6 pecmTap6] & [pa'pēme pe-1mape] \\
\hline 3 & Digo papima pimapa baixinho. & [papTi -m6 picmTap6] & [pa'pime pîmape] \\
\hline 4 & Digo papoma pomapa baixinho. & [papTo -m6 pocmTap6] & [pa'pōme po'mape] \\
\hline 5 & Digo papuma pumapa baixinho. & [papTu -m6 pucmTap6] & [pa'püme pu'tmape] \\
\hline \multicolumn{4}{|c|}{ Consoante nasal alveolar } \\
\hline 6 & Digo papana panapa baixinho. & [papT6 n6 pacnTap6] & [pa'pëne pe'nape] \\
\hline 7 & Digo papena penapa baixinho. & [papTe -n6 pecnTap6] & [pa'pēne pe-nape] \\
\hline 8 & Digo papina pinapa baixinho. & [papTi n6 picnTap6] & [pa'pine pinnape] \\
\hline 9 & Digo papona ponapa baixinho. & [papTo -n6 pocnTap6] & [pa'pōne po'nape] \\
\hline 10 & Digo papuna punapa baixinho. & [papTu -n6 pucnTap6] & [pa'püne pữnape] \\
\hline
\end{tabular}

Legenda: IPA - International Phonetic Alphabet; PB - português brasileiro.

Fonte: Adaptado de Vieira (2017, p. 128).

\footnotetext{
${ }^{2}$ Esta pesquisa foi aprovada pelo Comitê de Ética em Pesquisa com Seres Humanos (CEPSH) da Pró-Reitoria em Pesquisa e Extensão da UFSC, sob o processo número 2057 e Folha de Rosto (FR) número 434924.
} 
No Quadro 1, o sublinhado representa a sílaba tônica, enquanto o negrito indica a estrutura-alvo, constituída por vogal precedente + consoante nasal + vogal seguinte $\left(\mathrm{V}_{1} \mathrm{CnV}_{2}\right)$. No primeiro logatoma do par, o som-alvo está em contexto átono (pós-tônico) e, no segundo, em contexto tônico. Em ambos os contextos de tonicidade, o contexto vocálico precedente à consoante nasal é formado por uma das seguintes vogais do $\mathrm{PB}[\mathrm{i}$ e a o u].

Os dados de fala foram coletados em uma sala com tratamento acústico, por meio de instrumentos não invasivos de análise aerodinâmica: Estação EVA e piezoelétrico ${ }^{3}$. Esses instrumentos fazem parte da plataforma de estudos fisiológicos da fala, do Laboratoire de Phonétique et Phonologie da Université Paris III (França), e a coleta dos dados foi realizada no período de setembro de 2011 a março de 2012.

A gravação para cada instrumento foi realizada em dias diferentes e foi solicitado que cada participante repetisse três vezes os logatomas constituintes do corpus da pesquisa. As frases-veículo do corpus foram organizadas em uma sequência aleatória e apresentadas em slides no computador.

A Estação EVA permite analisar parâmetros de FAO, provenientes da cavidade oral; e de FAN, advindos da cavidade nasal, coletados por canais separados, mas simultaneamente, e em conjunto com um microfone oral que capta sinais acústicos orais. Nota-se, na Figura 2, o equipamento Estação EVA e a sua disposição no momento da coleta de dados.

Figura 2. Estação EVA: equipamento utilizado para a gravação de dados e sua disposição no momento da coleta de dados

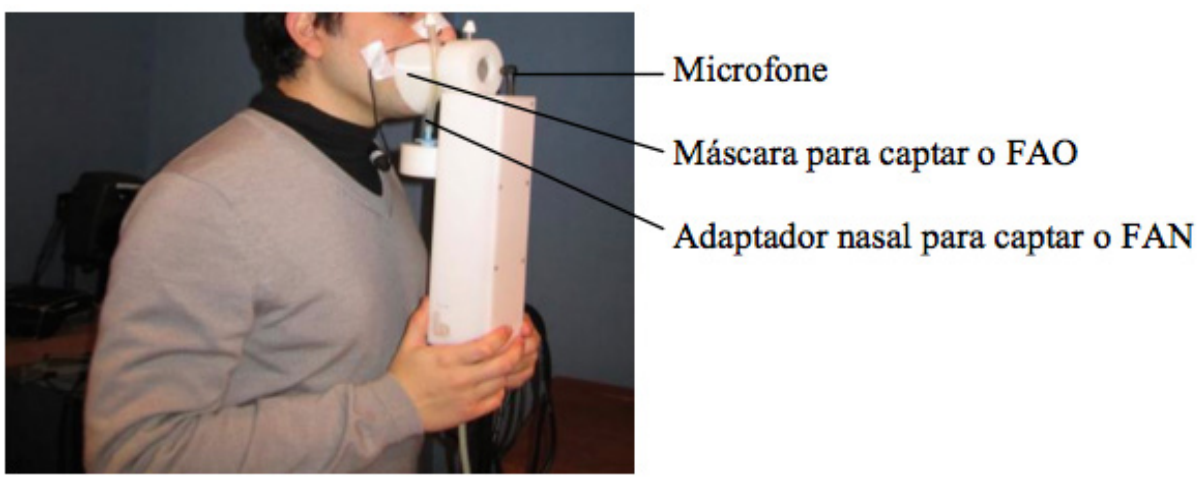

Legenda: FAN - fluxo de ar nasal; FAO - fluxo de ar oral.

Fonte: Arquivo pessoal da autora (2012).

O piezoelétrico, afixado na superfície externa do nariz, capta as vibrações nasais decorrentes somente da saída do fluxo aéreo da cavidade nasal, em conjunto com microfone oral permitindo também a análise acústica. Verifica-se, na Figura 3, o equipamento piezoelétrico e a sua disposição no momento da coleta de dados.

\footnotetext{
${ }^{3}$ Informações sobre esses equipamentos estão disponíveis em http://lpp.in2p3.fr/-Plateformephysiologique- $/$. Acesso em 16 de ago. de 2017.
} 
Figura 3. Piezoelétrico: equipamento utilizado para a gravação de dados e sua disposição no momento da coleta de dados

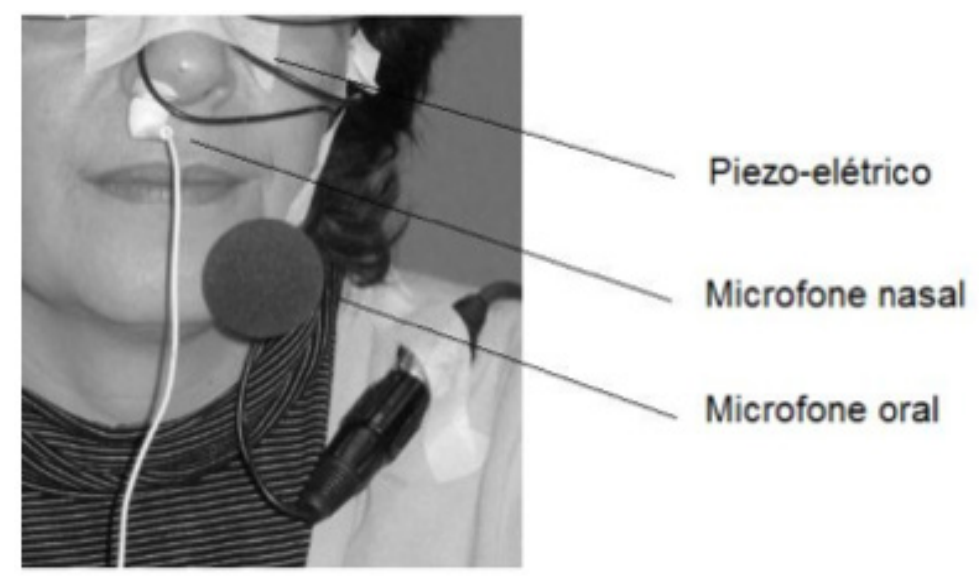

Fonte: Arquivo pessoal da autora (2012).

Foram utilizadas medidas sincronizadas de dados acústicos e aerodinâmicos, sendo possível gerar medidas de root means square (RMS) ${ }^{4}$ para todos os dados aerodinâmicos. Obtivemos 100 ocorrências com a Estação EVA, nas quais foram investigadas as configurações das curvas de FAN, de modo qualitativo. Contamos ainda com 206 ocorrências advindas do piezoelétrico, nas quais foram analisadas medidas de RMS das curvas FAN, com apoio de análises estatísticas.

Para verificar se havia diferença significativa entre os valores médios das medidas RMS da curva de FAN das consoantes [m] e [n], considerando o valor significativo de $p<$ 0,05, foi utilizada estatística não-paramétrica com os testes Mann-Whitney ou KruskallWallis, com o programa SPSS 5 , além do Post Hoc de Dunn.

\section{Resultados e discussão}

\subsection{Análise qualitativa: configurações do sinal de fluxo aéreo oral (FAO) e de fluxo aéreo nasal (FAN) obtidas a partir da Estação EVA}

$\mathrm{Na}$ análise aerodinâmica qualitativa, utilizamos os dados aerodinâmicos das consoantes nasais $[\mathrm{m}]$ e $[\mathrm{n}]$, coletados pela Estação EVA. Tal equipamento permitiu visualizar as curvas de FAO e de FAN, conforme indicado na Figura 4.

\footnotetext{
${ }^{4}$ Os sinais de vibração captados pelo piezoelétrico e pela Estação EVA consistem, geralmente, de inúmeras frequências que ocorrem simultaneamente. Com a utilização da técnica de análise de frequência, é possível relacionar a amplitude do sinal com a sua respectiva frequência. Uma das maneiras pela qual a amplitude da vibração pode ser quantificada é o nível quadrático médio ou root mean square (RMS).

${ }^{5}$ IBM Corp. Released 2011. IBM SPSS Statistics for Windows, Version 20.0. Armonk, NY: IBM Corp. http://www-01.ibm.com/support/docview.wss?uid=swg21476197.
} 
Figura 4. Canais nasal (FAN) e oral (FAO), coletados pela Estação EVA, indicando as curvas de FAN e FAO (em RMS) da região $\mathrm{V}_{1} \mathrm{CnV}_{2}$ em [m] (a) e [n] (b)

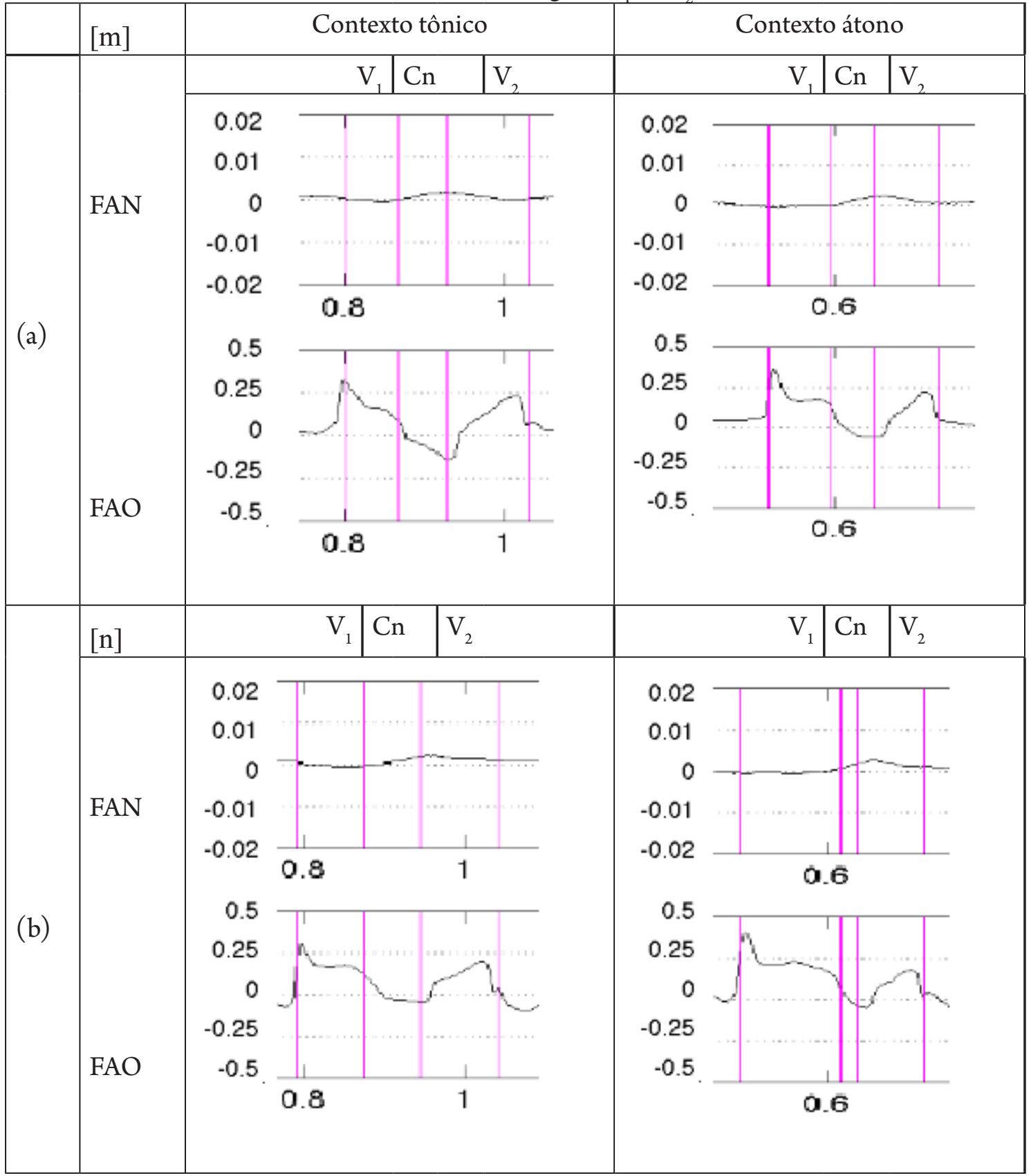

Legenda: FAN - fluxo aéreo nasal; FAO - fluxo aéreo oral; $\mathrm{V}_{1}$ - vogal precedente; $\mathrm{Cn}$ - consoante nasal ([m] ou $[\mathrm{n}]) ; \mathrm{V}_{2}$ - vogal seguinte.

Fonte: Dados primários (2018).

Conforme observamos na Figura 4 (a) e (b), as produções das vogais precedentes e seguintes às consoantes nasais [m] e [n] apresentaram curvas de FAO com amplitudes variadas, ao passo que as consoantes nasais $(\mathrm{Cn})$ praticamente não apresentaram amplitude de FAO. Essa parece ser uma característica comum da aerodinâmica de consoantes nasais $[\mathrm{m}] \mathrm{e}[\mathrm{n}]$. 
Podemos inferir a duração do fechamento labial - para [m], e a duração da oclusão da língua no palato - para [n], nas regiões das curvas do FAO, em que a amplitude é muito baixa. Esses intervalos de muito baixa amplitude indicam, portanto, a oclusão na passagem de ar pela cavidade oral nas duas consoantes nasais estudadas.

Já as curvas de FAN, segundo a Figura 4 (a) e (b), mostraram-se evidentes em ambas as consoantes nasais $([\mathrm{m}]$ e $[\mathrm{n}])$ e apresentaram variações dentre os contextos de tonicidade em que as consoantes estavam inseridas (átonos pós-tônicos - à direita e tônicos - à esquerda). Além disso, os contextos vocálicos precedentes apresentaram variadas curvas de FAN e as vogais seguintes às consoantes exibiram curvas de FAN em toda sua extensão com razoável amplitude.

Comparando-se as curvas de FAO e de FAN, podemos perceber que, durante o intervalo de muito baixa amplitude de FAO, o FAN tem aumento considerável na região consonantal e remanescências elevadas durante a vogal seguinte, indicando propagação do FAN na maioria dos dados. Também podemos destacar a presença da antecipação do FAN nas vogais precedentes às consoantes nasais $[\mathrm{m}] \mathrm{e}[\mathrm{n}]$. Esses resultados indicam que a nasalidade se inicia antes da consoante nasal, na vogal precedente e se estende após a consoante nasal, na vogal seguinte.

\subsection{Análise qualitativa: configurações de curvas de fluxo aéreo nasal (FAN) obtidas a partir do piezoelétrico}

Seguimos, então, com a investigação qualitativa por meio da inspeção visual das configurações das curvas de FAN com o software Praat ${ }^{6}$, obtidas a partir do piezoelétrico. Nessa análise qualitativa, buscamos identificar se as configurações das curvas de FAN eram semelhantes entre as duas consoantes nasais ou se indicariam diferenças entre elas.

Para isso, foram realizadas análises das configurações das curvas de FAN, considerando o som nasal consonantal e os contextos vocálicos adjacentes, que podem ser observadas na Figura 5 (a) e (b).

Figura 5. Formas-de-onda e configurações aerodinâmicas das curvas de FAN, coletadas pelo piezoelétrico, com destaque da região $\mathrm{V}_{1} \mathrm{CnV}_{2}$ nas consoantes nasais [m] e [n]

\footnotetext{
${ }^{6}$ Software utilizado em análise e síntese da fala, desenvolvido por Paul Boersma e David Weenink, do Institute of Phonetic Sciences, da University of Amsterdam. Disponível em http://www.fon. hum.uva.nl/. Acesso em 23 de ago. de 2017.
} 


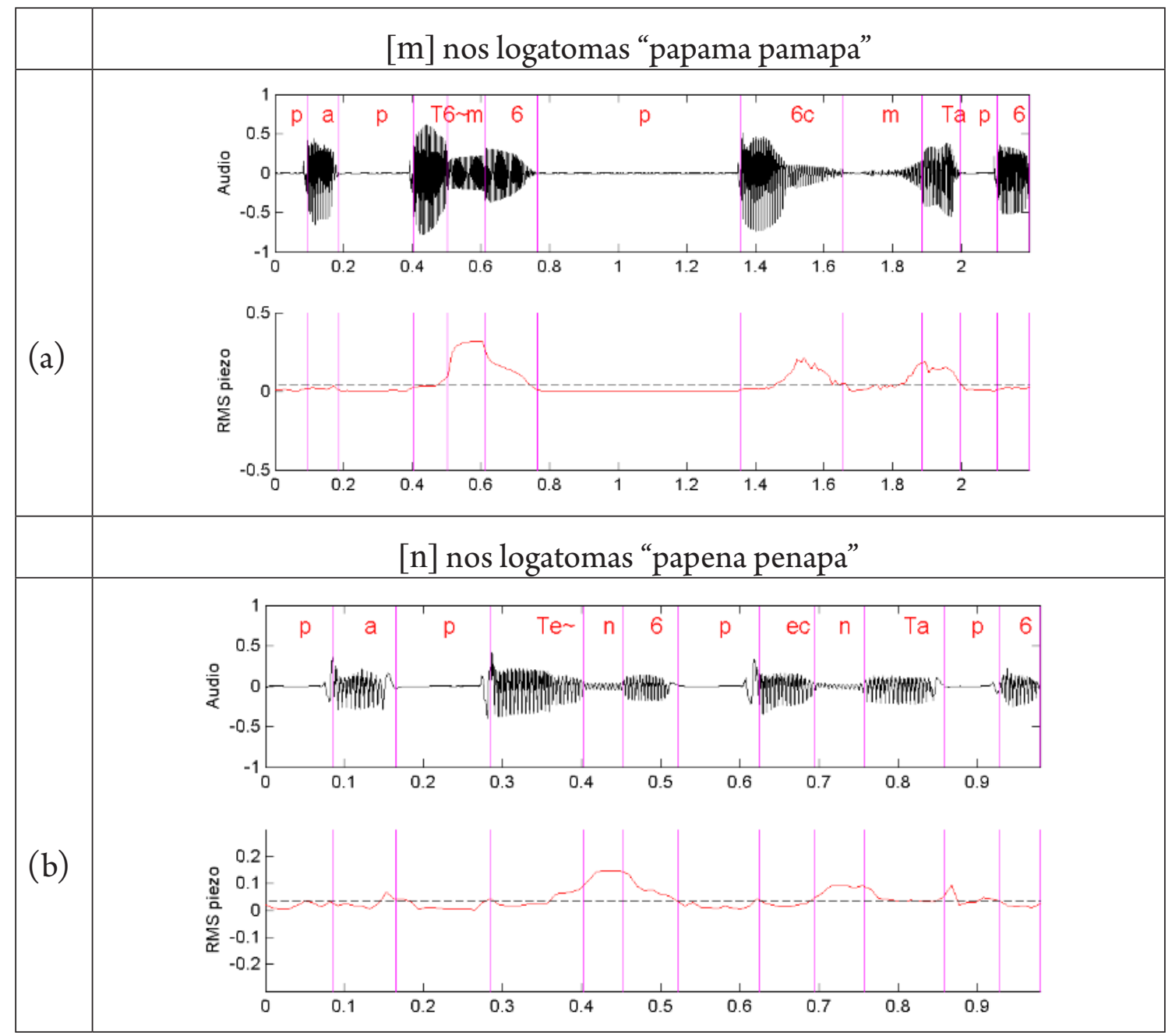

Legenda: FAN - fluxo aéreo nasal; $\mathrm{V}_{1}$ - vogal precedente; $\mathrm{Cn}$ - consoante nasal $([\mathrm{m}]$ ou $[\mathrm{n}]) ; \mathrm{V}_{2}-$ vogal seguinte.

Fonte: Dados primários (2018).

Os resultados aerodinâmicos indicaram que as curvas de FAN tiveram, na maior parte dos dados, as mesmas configurações para [m] e [n], conforme ilustrado na Figura 5 (a) e (b), respectivamente. Verificamos uma configuração em platô preponderante de curva de FAN em 92\% das produções, como, por exemplo, a apresentada na região da consoante nasal (Cn) na Figura 5(a) e (b). Foram observadas ainda outras variações assimétricas pouco representativas em $8 \%$ das produções. Embora, na inspeção visual, considerando todos os dados analisados, não tenhamos constatado diferenças evidentes entre as consoantes nasais [m] e [n] quanto às curvas de FAN, alguns dados, como os da Figura 5 (a) e (b), indicaram que o FAN, na produção de [m], mostra diferenças de antecipação da nasalização entre a posição átona e a tônica. Na posição átona, foi mais significativo, apresentando uma antecipação de nasalidade moderada e, na posição tônica, a antecipa- 
ção de nasalidade ocorre próximo ao onset de $[\mathrm{m}]$, mostrando pouca antecipação sobre a vogal antecedente. No entanto, o FAN na produção do [n] não mostrou diferenças entre as posições átona e tônica.

Ainda, se observarmos o comportamento das duas consoantes quando estão em posição tônica, veremos diferenças de comportamento do FAN que, na produção de [m], exibe pouca antecipação sobre a vogal antecedente; enquanto, na produção de [n], apresenta antecipação moderada. Na pesquisa de Mendonça e Seara (2015), já havia sido observado também que a qualidade da consoante nasal influencia o grau de nasalização da vogal que a antecede. As autoras verificaram que as vogais antecedentes são nasalizadas, havendo diferença no início do FAN entre as consoantes nasais, conforme observado anteriormente para a posição tônica.

A fim de confirmarmos esses dados qualitativos, foi realizada uma análise quantitativa com auxílio da estatística, considerando as medidas RMS coletadas em pontos (mínimos, médios, máximos, do início, do meio e do fim) ao longo da curva de FAN. Esta análise é apresentada a seguir.

\subsection{Análise quantitativa: parâmetros aerodinâmicos com base em medidas de RMS advindas dos dados exibidos pelo piezoelétrico}

Nas análises quantitativas, foram consideradas medidas RMS relativas aos valores mínimos (1), médios (2), máximos (3), do início (4), do meio (5) e do fim (6) das curvas de FAN, conforme se vê na Figura 6, a seguir.

Figura 6. Pontos de coleta dos valores da curva de FAN (em RMS): mínimo (1), médio (2), máximo (3), do início (4), do meio (5) e do fim (6)

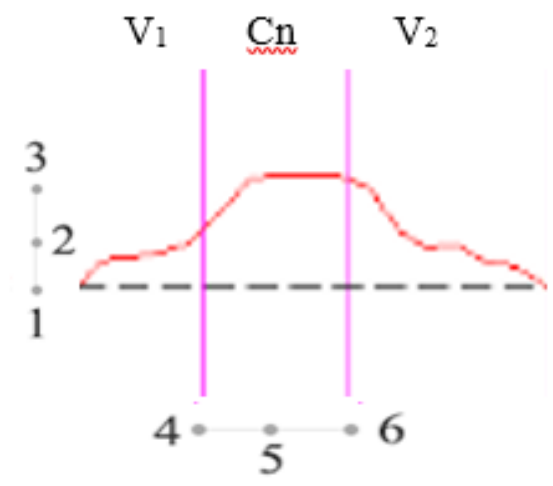

Legenda: FAN - fluxo aéreo nasal; RMS - Root Means Square; $\mathrm{V}_{1}$ - vogal precedente; $\mathrm{Cn}$ - Som-alvo; $\mathrm{V}_{2}$ - vogal seguinte.

Fonte: Dados primários (2018).

Na Tabela 1, estão expostos os valores médios e seus correspondentes desvios pa- 
drão (dp) das curvas de FAN, advindos do piezoelétrico, considerando o sexo feminino e masculino separadamente, para as consoantes $[\mathrm{m}] \mathrm{e}[\mathrm{n}]$.

Tabela 1. Valores médios mínimos, médios, máximos, do início, do meio e do fim das medidas da curva de FAN (em RMS) nas consoantes nasais [m] e [n], separados por sexo

\begin{tabular}{|l|c|c|c|c|c|}
\hline & \multicolumn{2}{|c|}{$[\mathbf{m}]$} & \multicolumn{2}{|c|}{ [n] } & \\
\hline Feminino & média & $\mathrm{dp}$ & média & $\mathrm{dp}$ & $\mathrm{p}$ valor \\
\hline Mínimos & 0,069 & 0,035 & 0,080 & 0,037 & 0,071 \\
\hline Médios & 0,108 & 0,035 & 0,117 & 0,034 & 0,137 \\
\hline Máximos & 0,131 & 0,038 & 0,137 & 0,037 & 0,426 \\
\hline Do início & 0,096 & 0,034 & 0,109 & 0,038 & 0,058 \\
\hline Do meio & 0,117 & 0,041 & 0,127 & 0,040 & 0,162 \\
\hline Do fim & 0,120 & 0,042 & 0,130 & 0,039 & 0,214 \\
\hline Masculino & & & & & \\
\hline Mínimos & 0,109 & 0,072 & 0,126 & 0,048 & $0,032 *$ \\
\hline Médios & 0,182 & 0,077 & 0,194 & 0,061 & 0,405 \\
\hline Máximos & 0,227 & 0,081 & 0,239 & 0,072 & 0,366 \\
\hline Do início & 0,184 & 0,083 & 0,194 & 0,067 & 0,736 \\
\hline Do meio & 0,197 & 0,082 & 0,209 & 0,074 & 0,551 \\
\hline Do fim & 0,200 & 0,087 & 0,215 & 0,075 & 0,348 \\
\hline
\end{tabular}

Legenda: * - valor significativo ( $\mathrm{p}<0,05)$; dp - desvio padrão; FAN - fluxo aéreo nasal.

Fonte: Dados primários (2018).

Os resultados mostraram que, para o sexo masculino, houve diferença estatística entre as consoantes $[\mathrm{m}] \mathrm{e}[\mathrm{n}]$ somente entre os valores mínimos (1) da curva de FAN ( $p$ $=0,032)$. Nos demais parâmetros aerodinâmicos observados nas curvas de FAN - médios (2), máximos (3), do início (4), do meio (5) e do fim (6) -, essas consoantes não se diferenciaram. Isso está de acordo com o resultado da análise qualitativa na qual as formas-de-onda e as configurações de curvas de fluxo aéreo nasal entre $[\mathrm{m}]$ e $[\mathrm{n}]$ pareciam não se diferir de modo evidente.

Agora serão abordados os resultados obtidos quanto aos parâmetros aerodinâmicos investigados em função da tonicidade das sílabas em que se encontravam as consoantes nasais. 


\subsubsection{Contexto de tonicidade}

Quanto ao contexto de tonicidade, inicialmente, na análise qualitativa, verificamos medidas RMS mais elevadas na curva de FAN dos contextos átonos (pós-tônicos) do que nos tônicos para a maioria dos dados das nasais [m] e [n], conforme Tabela 2.

Tabela 2. Valores médios mínimos, médios, máximos, do início, do meio e do fim das medidas da curva de FAN (em RMS) por contexto de tonicidade (átono e tônico) entre as consoantes nasais $[\mathrm{m}]$ e $[\mathrm{n}]$, separados por sexo

\begin{tabular}{|l|c|c|c|c|c|c|c|c|c|c|}
\hline & \multicolumn{4}{|c|}{$[\mathbf{m}]$} & \multicolumn{5}{c|}{ [n] } \\
\hline Feminino & \multicolumn{2}{|c|}{ átono } & \multicolumn{2}{|c|}{ tônico } & & \multicolumn{2}{c|}{ átono } & \multicolumn{2}{c|}{ tônico } & \\
\hline Mínimos & 0,088 & 0,035 & 0,050 & 0,023 & $<0,001^{*}$ & 0,103 & 0,032 & 0,058 & 0,027 & $<0,001^{*}$ \\
\hline Médios & 0,126 & 0,032 & 0,090 & 0,027 & $<0,001^{*}$ & 0,136 & 0,028 & 0,098 & 0,029 & $<0,001^{*}$ \\
\hline Máximos & 0,149 & 0,038 & 0,113 & 0,030 & $<0,001^{*}$ & 0,155 & 0,032 & 0,118 & 0,031 & $<0,001^{*}$ \\
\hline Do início & 0,111 & 0,031 & 0,081 & 0,031 & $<0,001^{*}$ & 0,127 & 0,030 & 0,090 & 0,036 & $<0,001^{*}$ \\
\hline Do meio & 0,135 & 0,039 & 0,098 & 0,034 & $<0,001^{*}$ & 0,148 & 0,034 & 0,107 & 0,034 & $<0,001^{*}$ \\
\hline Do fim & 0,140 & 0,042 & 0,101 & 0,033 & $<0,001^{*}$ & 0,149 & 0,034 & 0,111 & 0,033 & $<0,001^{*}$ \\
\hline Masculino & média & $\mathrm{dp}$ & média & $\mathrm{dp}$ & $\mathrm{p}$ valor & média & $\mathrm{dp}$ & média & dp & p valor \\
\hline Mínimos & 0,134 & 0,082 & 0,083 & 0,051 & $0,014^{*}$ & 0,135 & 0,043 & 0,116 & 0,052 & 0,234 \\
\hline Médios & 0,203 & 0,085 & 0,161 & 0,063 & 0,085 & 0,194 & 0,059 & 0,195 & 0,065 & 0,946 \\
\hline Máximos & 0,236 & 0,094 & 0,217 & 0,067 & 0,469 & 0,231 & 0,069 & 0,246 & 0,076 & 0,521 \\
\hline Do início & 0,205 & 0,085 & 0,164 & 0,077 & 0,118 & 0,187 & 0,061 & 0,201 & 0,074 & 0,517 \\
\hline Do meio & 0,216 & 0,089 & 0,178 & 0,071 & 0,142 & 0,207 & 0,072 & 0,210 & 0,078 & 0,839 \\
\hline Do fim & 0,220 & 0,095 & 0,181 & 0,076 & 0,161 & 0,215 & 0,073 & 0,216 & 0,079 & 0,935 \\
\hline
\end{tabular}

Legenda: * - valor significativo $(\mathrm{p}<0,05)$ verificado com estatística não paramétrica com teste Mann-Whitney; dp - desvio padrão; FAN - fluxo aéreo nasal.

Fonte: Adaptado de Vieira (2017, p. 278-279).

Para os participantes do sexo feminino, segundo a Tabela 2, verificamos diferenças significativas entre o contexto átono e tônico, para todos os parâmetros aerodinâmicos analisados, tanto em [m] quanto em [n]. No que se refere aos participantes do sexo masculino, houve diferença significativa somente entre os valores mínimos (1) da curva de FAN e apenas para a consoante [m].

Esses resultados podem estar relacionados a questões de diferenças de entoação e ritmo de fala entre homens e mulheres. As produções dos logatomas realizadas pelas 
mulheres exibiram maior ênfase e maior tempo na emissão dos sons-alvo em contexto átono (pós-tônico), ou seja, maior duração da consoante nasal em sílaba final, levando a um maior fluxo aéreo nasal nesta posição da palavra do que em sílaba medial. Ainda, esses diferentes resultados entre o sexo masculino e feminino corroboram estudos do PB sobre características aerodinâmicas de sons nasais (MARINO et al., 2016; MENDONÇA; SEARA, 2015). O estudo de Marino et al. (2016) indicou valores de nasalância mais altos para mulheres quando comparados aos dos homens, particularmente para os adultos.

Ainda, outra possível explicação para as consoantes nasais [m] e [n] átonas (Ex.: papema) apresentarem valores de FAN mais altos, evidenciando, ao menos para o sexo feminino, maior nasalização do que as consoantes nasais tônicas (Ex.: pemapa), pode ser pelo fato de que, quando a consoante nasal ocupa o contexto átono (pós-tônico), a vogal tônica que a antecede nasaliza-se (nasalização regressiva), e isso causaria um aumento da nasalidade, e uma duração maior da abertura do véu. Esse espraiamento de nasalidade sobre a vogal antecedente atingiria assim um nível mais significativo de nasalização. Já quando a consoante nasal ocupa o contexto tônico, a nasalização praticamente começa nela, e por essa razão parece não atingir um FAN tão expressivo.

Passaremos aos resultados quanto ao contexto de vogal precedente e sua influência nos parâmetros aerodinâmicos aqui investigados.

\subsubsection{Contexto vocálico precedente}

As características descritas na análise qualitativa das configurações das curvas de FAN pareceram, em um primeiro momento, ser independentes da vogal precedente, ou seja, indicando que o tipo da configuração não estava associado a alguma vogal específica que antecedesse as consoantes nasais em estudo.

Os resultados obtidos com relação aos parâmetros aerodinâmicos investigados para a consoante nasal [m], levando em conta os diferentes contextos vocálicos precedentes e o sexo, encontram-se na Tabela 3.

Tabela 3. Valores médios mínimos, médios, máximos, do início, do meio e do fim das medidas RMS da curva de FAN por contexto vocálico precedente, considerando a consoante nasal [m], separados por sexo 


\begin{tabular}{|c|c|c|c|c|c|c|c|c|c|c|c|}
\hline [m] & \multicolumn{2}{|c|}{ 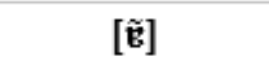 } & \multicolumn{2}{|c|}{ [ẽ] } & \multicolumn{2}{|c|}{ [i] } & \multicolumn{2}{|c|}{ [थ̃] } & \multicolumn{2}{|c|}{$[\tilde{\mathbf{u}}]$} & \multirow[b]{2}{*}{$\mathrm{p}$ valor } \\
\hline Feminino & média & $d p$ & média & $d p$ & média & $d p$ & média & $d p$ & média & $d p$ & \\
\hline Mínimos & 0,057 & 0,031 & 0,059 & 0,032 & 0,080 & 0,042 & 0,065 & 0,030 & 0,083 & 0,038 & 0,323 \\
\hline Médios & 0,096 & 0,038 & 0,107 & 0,035 & 0,118 & 0,036 & 0,108 & 0,031 & 0,113 & 0,038 & 0,669 \\
\hline Máximos & 0,122 & 0,041 & 0,129 & 0,038 & 0,139 & 0,037 & 0,133 & 0,039 & 0,131 & 0,042 & 0,867 \\
\hline Do início & 0,082 & 0,027 & 0,098 & 0,036 & 0,104 & 0,033 & 0,093 & 0,038 & 0,105 & 0,036 & 0,552 \\
\hline Do meio & 0,103 & 0,048 & 0,118 & 0,039 & 0,127 & 0,041 & 0,113 & 0,037 & 0,122 & 0,043 & 0,722 \\
\hline Do fim & 0,105 & 0,049 & 0,121 & 0,040 & 0,128 & 0,042 & 0,125 & 0,040 & 0,122 & 0,043 & 0,726 \\
\hline Masculino & média & $\mathrm{dp}$ & média & $d p$ & média & $d p$ & média & $\mathrm{dp}$ & média & $\mathrm{dp}$ & $\mathrm{p}$ valor \\
\hline Mínimos ${ }^{€}$ & 0,082 & 0,039 & 0,094 & 0,040 & 0,193 & 0,092 & 0,055 & 0,034 & 0,121 & 0,061 & $0,002^{*}$ \\
\hline Médios & 0,156 & 0,072 & 0,176 & 0,051 & 0,238 & 0,085 & 0,139 & 0,084 & 0,203 & 0,062 & 0,118 \\
\hline Máximos & 0,203 & 0,074 & 0,221 & 0,067 & 0,273 & 0,092 & 0,192 & 0,095 & 0,244 & 0,064 & 0,186 \\
\hline Do início ${ }^{€}$ & 0,163 & 0,082 & 0,161 & 0,042 & 0,259 & 0,078 & 0,130 & 0,093 & 0,209 & 0,058 & $0,024^{*}$ \\
\hline Do meio & 0,170 & 0,085 & 0,193 & 0,057 & 0,243 & 0,078 & 0,153 & 0,094 & 0,226 & 0,076 & 0,177 \\
\hline Do fim & 0,174 & 0,089 & 0,207 & 0,068 & 0,241 & 0,081 & 0,159 & 0,105 & 0,220 & 0,083 & 0,375 \\
\hline
\end{tabular}

Legenda: * - valor significativo $(p<0,05)$ verificado com estatística não paramétrica com teste Kruskall-Wallis; dp - desvio padrão; FAN - fluxo aéreo nasal; $€$ - diferença entre $[i)]$ e $[0)]$.

Fonte: Adaptado de Vieira (2017, p. 282-283).

$\mathrm{Na}$ Tabela 4, são apresentados os resultados referentes à consoante nasal [n], obtidos com relação aos parâmetros aerodinâmicos analisados, considerando os diferentes contextos vocálicos precedentes e o sexo.

Tabela 4. Valores médios mínimos, médios, máximos, do início, do meio e do fim das medidas RMS da curva de FAN por contexto vocálico precedente, considerando a consoante nasal $[\mathrm{n}]$, separados por sexo

\begin{tabular}{|c|c|c|c|c|c|c|c|c|c|c|c|}
\hline [n] & \multicolumn{2}{|c|}{$[\tilde{\mathbf{\varepsilon}}]$} & \multicolumn{2}{|c|}{ [ẽ] } & \multicolumn{2}{|c|}{ [i] } & \multicolumn{2}{|c|}{ [थ̃] } & \multicolumn{2}{|c|}{$[\tilde{\mathbf{u}}]$} & \\
\hline Feminino & média & $\mathrm{dp}$ & média & $\mathrm{dp}$ & média & $d p$ & média & $d p$ & média & $d p$ & $\mathrm{p}$ valor \\
\hline Mínimos $^{£, \beta}$ & 0,072 & 0,036 & 0,059 & 0,031 & 0,101 & 0,038 & 0,076 & 0,030 & 0,100 & 0,036 & $0,019^{*}$ \\
\hline Médios & 0,122 & 0,038 & 0,102 & 0,029 & 0,121 & 0,032 & 0,117 & 0,034 & 0,124 & 0,037 & 0,503 \\
\hline Máximos & 0,146 & 0,042 & 0,127 & 0,033 & 0,135 & 0,032 & 0,137 & 0,039 & 0,138 & 0,038 & 0,742 \\
\hline Do início & 0,111 & 0,042 & 0,085 & 0,039 & & & & 0,036 & & 36 & \\
\hline Do meio & 0,134 & 0,046 & 0,113 & 0,035 & 0,131 & 0,036 & 0,129 & 0,042 & 0,131 & 0,042 & 0,673 \\
\hline Do fim & 0,141 & 0,045 & 0,120 & 0,033 & 0,128 & 0,036 & 0,130 & 0,039 & 0,130 & 0,042 & 0,746 \\
\hline Masculino & média & $\mathrm{dp}$ & média & $d p$ & média & $\mathrm{dp}$ & média & $\mathrm{dp}$ & média & $\mathrm{dp}$ & $\mathrm{p}$ valor \\
\hline Mínimos $^{\alpha}$ & 0,098 & 0,033 & 0,102 & 0,033 & 0,151 & 0,059 & 0,115 & 0,029 & 0,163 & 0,049 & $0,039^{*}$ \\
\hline Médios & 0,179 & 0,055 & 0,160 & 0,035 & 0,226 & 0,078 & 0,184 & 0,051 & 0,223 & 0,064 & 0,186 \\
\hline Máximos & 0,232 & 0,077 & 0,205 & 0,054 & 0,262 & 0,082 & 0,233 & 0,076 & 0,262 & 0,068 & 0,368 \\
\hline Do início $^{\beta}$ & 0,171 & 0,059 & 0,142 & 0,044 & 0,227 & 0,076 & 0,177 & 0,028 & 0,252 & 0,065 & $0,012^{*}$ \\
\hline Do meio & 0,199 & 0,074 & 0,164 & 0,053 & 0,249 & 0,089 & 0,195 & 0,066 & 0,237 & 0,068 & 0,092 \\
\hline Do fim & 0,212 & 0,075 & 0,182 & 0,052 & 0,245 & 0,095 & 0,214 & 0,079 & 0,224 & 0,075 & 0,589 \\
\hline
\end{tabular}


Legenda: * - valor significativo $(\mathrm{p}<0,05)$ verificado com estatística não paramétrica com teste Kruskall-Wallis; dp - desvio padrão; FAN - fluxo aéreo nasal; $£$ - diferença entre $[\varepsilon)]$ e $[i)] ; \beta$ - diferença entre $[\varepsilon)]$ e $[v)] ; \alpha$ - diferença entre $[(E)]$ e $[v)]$.

Fonte: Adaptado de Vieira (2017, p. 282-283).

Com base em estatística não-paramétrica com teste Kruskall-Wallis e o Post Hoc de Dunn, observamos que, para o sexo feminino, conforme Tabela 4, a consoante [n] apresentou diferenças significativas $(p<0,05)$ nos valores mínimos entre as vogais $[\varepsilon)] /$ $[i)]$ e $[\varepsilon)] /[v)]$. Para o sexo masculino, os valores mínimos e do início da curva de FAN foram os mais influenciados pelos contextos vocálicos, com diferenças significativas entre as vogais precedentes: $[\mathrm{i})] /[\mathrm{o})],[\varepsilon)] /[\mathrm{v})] \mathrm{e}[\mathrm{E})] /[\mathrm{v})]$, para as duas consoantes $[\mathrm{m}] \mathrm{e}[\mathrm{n}]$, conforme dados exibidos nas Tabelas 3 e 4, respectivamente.

De acordo com esses resultados, na análise aerodinâmica quantitativa, o contexto vocálico precedente às consoantes nasais mostrou influência significativa em poucos parâmetros, ou seja, somente nos valores mínimos (1) e do início (4) da curva de FAN (conforme Figura 6 - Seção 4.3). Esse último parâmetro aerodinâmico está relacionado, justamente, com o ponto da curva de FAN mais próximo à vogal precedente (à esquerda da curva) e o primeiro aos menores valores em amplitude das curvas de FAN.

Tal resultado indica, portanto, que o início da curva de FAN nas consoantes nasais ([m] e $[\mathrm{n}])$, onde ocorre também a menor amplitude da saída de FAN, caracteriza-se como a região que recebe influência da qualidade vocálica precedente.

\subsection{Perspectiva dinâmica da fala}

O estudo de Oliveira e Teixeira (2007) sobre os gestos das consoantes nasais no português europeu contribuiu para fazermos inferências articulatórias com as configurações aerodinâmicas descritas nas curvas de FAN das consoantes nasais no presente estudo. Como visto anteriormente (Seção 2), essas autoras verificaram que o gesto vélico para as consoantes nasais $[\mathrm{m}]$ e $[\mathrm{n}]$ estava constituído por três tempos: abertura, platô e fechamento, o que aponta uma consonância com nossos resultados aerodinâmicos qualitativos (Seção 4.1 e 4.2) e quantitativos (Seção 4.3) que, em sua maioria, não apresentaram diferenças entre as consoantes $[\mathrm{m}]$ e $[\mathrm{n}]$ para os parâmetros aerodinâmicos investigados, à exceção dos valores mínimos.

Assim, supomos que, quando a curva de FAN está aumentando, o esfíncter velofaríngeo (EVF) está abrindo (abertura do gesto vélico); quando a curva de FAN está em 
platô, o EVF está aberto (platô do gesto vélico) e, quando a curva de FAN está decrescendo, o EVF está fechando (fechamento do gesto vélico).

Dessa maneira, ao apontarmos para a natureza dinâmica das consoantes nasais do $\mathrm{PB}$ e com base nas configurações aerodinâmicas descritas para [m] e [n], podemos inferir que haja a manutenção da abertura do movimento velar durante o platô.

\section{Considerações finais}

O presente artigo se propôs a investigar características aerodinâmicas da produção das consoantes nasais [m] e [n]. Com base nos resultados obtidos, confirmamos nossa hipótese inicial de que essas consoantes apresentam características semelhantes de FAO e não constatamos, em termos de FAN, que consoantes nasais [m] se diferenciam das [n].

Dessa forma, podemos considerar que não foi verificada distinção nos parâmetros do fluxo aéreo, comparando-se as consoantes nasais [m] e [n], nos tipos de configurações das curvas de FAO e FAN. Quanto aos valores mínimos, médios, máximos, iniciais, mediais e finais das curvas de FAN, a análise quantitativa demonstrou que as consoantes nasais $[\mathrm{m}]$ e $[\mathrm{n}]$ se diferenciaram, nos falantes masculinos, quanto aos valores aerodinâmicos 'míninos' das curvas de FAN, não indicando outros pontos de distinção aerodinâmica entre elas.

Verificamos, também, que as medições do FAN representaram uma indicação indireta do grau de abertura velofaríngea. E, por esse caminho, inferências articulatórias do movimento de abertura e fechamento do esfíncter velofaríngeo podem ser decorrentes dos resultados aerodinâmicos.

Na presente pesquisa, os resultados indicaram, para ambos os sexos:

(i) Quanto às configurações de curvas de fluxo aéreo oral (FAO) e de fluxo aéreo nasal (FAN) advindas da Estação EVA, movimentos semelhantes correspondentes às consoantes nasais $[\mathrm{m}] \mathrm{e}[\mathrm{n}]$. As curvas de FAO se assemelharam entre as consoantes nasais [m] e [n], apresentando muito baixa amplitude, o que indica oclusão na passagem de ar pela cavidade oral. $O$ formato das curvas de FAN mostrou configuração plana em sua extensão (em platô), destacando-se por apresentar maior amplitude em relação às vogais adjacentes.

(ii) Os valores médios, máximos, do início, do meio e do fim da curva de FAN, obtidos a partir do piezoelétrico, quando comparados entre as consoantes nasais [m] e [n], não apresentaram diferenças estatísticas entre a maior parte dos parâmetros avaliados. Essas consoantes se diferenciaram somente pelo 
parâmetro aerodinâmico obtido no ponto mínimo da curva de FAN.

(iii) Constatamos diferenças significativas nos parâmetros aerodinâmicos, por contexto de tonicidade e de vogal precedente, nas consoantes nasais [m] e [n]. Entretanto, isso ocorreu somente para alguns valores da curva de FAN (mínimos e do início), variando a consoante nasal influenciada por esses contextos e, ainda, de modo distinto entre os sexos.

(iv) Pelo viés da perspectiva dinâmica da fala, foi possível inferir aspectos articulatórios por meio de parâmetros aerodinâmicos. O comportamento aerodinâmico do FAN na produção das consoantes [m] e [n] permitiu a inferência articulatória de que o gesto vélico dessas consoantes seja formado por três tempos: abertura, platô (manutenção da abertura) e fechamento.

Este estudo na área das Ciências da Fala apresenta indícios de que a análise aerodinâmica contribui para um embasamento teórico na perspectiva dinâmica de produção da fala. Demonstra, ainda, as contribuições do dado fonético aliado às tecnologias dos instrumentos de análise aerodinâmica, promovendo um olhar mais detalhado que pode ser promissor nas áreas da Fonoaudiologia e da Linguística.

\section{Agradecimentos}

À equipe de pesquisadores do Laboratoire de Phonétique et Phonologie, da Université Paris III - Sorbonne Nouvelle, em Paris/França, especialmente à professora Jacqueline Vaissière e à Angelique Amelot.

Ao CNPq pela bolsa de produtividade.

Ao Programa de Bolsas Universitárias de Santa Catarina - UNIEDU/FUMDES, da Diretoria de Educação Superior da Secretaria de Estado da Educação.

Aos pareceristas desta Revista pela leitura cuidadosa e pelas contribuições para uma versão mais adequada deste artigo. No entanto, possíveis inadequações que porventura tenham permanecido são de responsabilidade dos autores.

\section{Referências}

AMELOT, A.; MICHAUD, A. Effets aérodynamiques du mouvement du velum: le cas des voyelles nasales du français. Actes des XXVI Journées d'Etudes sur la Parole. Halshs-00137628, version 1, v. 26, Mar., 2007.

BROWMAN, C.; GOLDSTEIN, L. Articulatory Phonology: an overview. In: Phonetica, v. 49, p. 155-180, 1992. 
COHN, A. C.; FOUGERON, C.; HUFFMAN, M. K. The Oxford Handbook of Laboratory Phonology. Oxford University Press: Oxford, 2012.

FOWLER, C. Coarticulation and theories of extrinsic timing control. Journal of Phonetics, v. 8, p. 113-133, 1980.

MARINO, V. C. C. et al. Valores de nasalância para sílabas produzidas por falantes do Português Brasileiro. CoDAS, São Paulo, v. 28, n. 3, p. 278-283, 2016. Disponível em: http://www.scielo.br/pdf/codas/v28n3/2317-1782-codas-2317-178220162015166. pdf. Acesso em 13 de abr. de 2018.

MEDEIROS, B. R.; D’IMPERIO, M.; ESPESSER, R. O apêndice nasal: dados aerodinâmicos e duracionais. Revista do GEL, São Paulo, v. 5, n. 2, p. 123-138, 2008.

MENDONÇA, C. S. I. de; SEARA, I. C. Análise aerodinâmica da nasalidade coarticulatória no falar florianopolitano. Revista Domínios de Linguagem, v. 9, n. 5, p. 83104, 2015. Disponível em: http://www.seer.ufu.br/index.php/dominiosdelinguagem/ article/view/29058. Acesso em 13 abr. 2018.

OLIVEIRA C.; TEIXEIRA, A. On gestures timing in european portugueses nasals. ICPhS XVI, Saarbrücken. p. 405-408, 2007.

SILVA, A. H. P.; ALVES, U. K. Editorial. GRADUS - Revista Brasileira de Fonologia de Laboratório, v. 1, n. 1, dez. 2016, p. 6-11. Disponível em: https://gradusjournal.com/ index.php/gradus/issue/view/1/Edi\%C3\%A7\%C3\%A3o\%20completa. Acesso em 13 abr. 2018.

VAISSIÈRE, J. Nasalité et phonétique: Le voile du palais et la parole. Conférence Tutorielle Invitée, Colloque sur le voile pathologique, Lyon, publié par la Société Française d'Acoustique, 1995.

VIEIRA, M. G. Estudo acústico e aerodinâmico das consoantes nasais do português brasileiro: variedade de florianópolis.2017. Tese (Doutorado em Linguística) - Universidade Federal de Santa Catarina. Florianópolis, 2017. Disponível em: https://repositorio.ufsc.br/ xmlui/bitstream/handle/123456789/182710/348938.pdf? sequence $=1$ \&isAllowed $=y$. Acesso em: 13 abr. 2018.

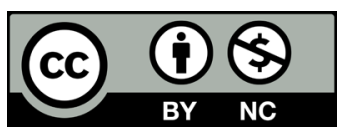

Data de submissão: 29/10/2017

Data de aceite: $21 / 02 / 2018$ 